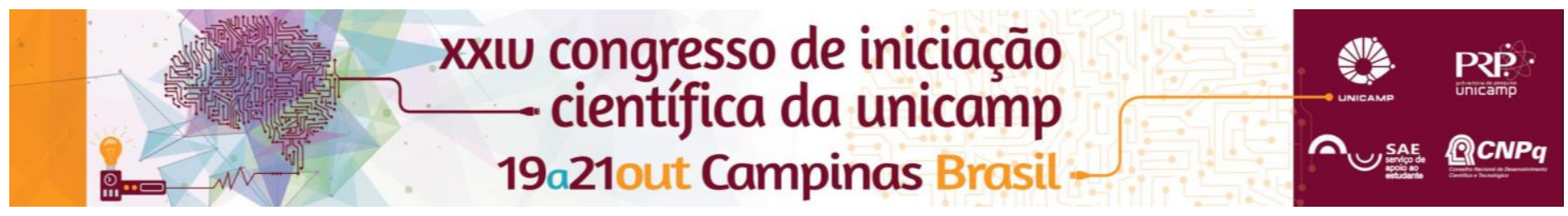

\title{
Evaluation of the degradation products of fibers elastics in Fibrillin-1 deficient cells.
}

\author{
Marina R. P. Garcia*, Guilherme G. Braga, Camila W. Leme, José C. Novello, Cristina P. Vicente, Cláudio C. \\ Werneck.
}

\begin{abstract}
Marfan syndrome (MSF) is a genetic disease related to the mutation of the gene encoding fibrillin-1, an extracellular matrix protein and a major component of elastic fiber microfibrils. Recent studies suggested that mutations in the fibrillin-1 gene increases the elastic fibers degradation. To clarify the products of that degradation, dermal fibroblasts were isolated from FBN-1 homozygote and wild type mices and were cultivated. Deposition of the components of the elastic fibers was assessed by methods of immunofluorescence and its degradation products were evaluate by gel-filtrations chromatography, SDS-page electrophoresis and by Western blot analysis. Immunofluorescence results revealed that in deficient FBN1 cells the deposition of MAGP-1, tropoelastin and fibrillin-1 was decreased. The methodology used to evaluate the degradation products of those cells do not allowed the visualization of proteins, except albumin. With some changes in the protocol used, as higher concentration of ammonium sulfate for the protein precipitation and higher concentration of acrylamide in electrophoresis SDS-page, those protein could have been acessed.
\end{abstract}

\section{Key words:}

Elastic fibers, fibrillin-1, Marfan syndrome.

\section{Introduction}

Marfan syndrome (MSF) is an autosomal dominant connective tissue disorder that has ocular, pulmonary and cardiovascular manifestations, which is related to fibrillin1 gene mutations. Fibrillin-1 is a high molecular weight protein $(\approx 350 \mathrm{kDa})$, component of the microfibril network of the extracellular matrix, which functions as a scaffold to the deposition of the elastic fibers, performing a structural role. The symptoms of the syndrome have been related to the dysregulation of the Transformation Growth Factor Beta (TGF- $\beta$ ). Recent studies have investigated the reduction of components of the elastic fibers in the extracellular matrix of fibrillin-1 deficient cells. The present study aims to evaluate the degradation products of elastic fibers in FBN-1 deficient cells.

\section{Results and Discussion}

Immunofluorescence was performed in wild type and FBN-1 deficient cells, using anti-mouse MAGP-1, fibrillin2 and fibrillin-1 antibodies. Decreased deposition of $83 \%$ in MAGP-1 (A, D and G), 87\% for tropoelastin (B, E and $H$ ), and $86 \%$ for fibrillin-1 (C, F and I) was observed when compared fibrillin-1 deficient cells with wild type cells.

Recent studies reveal a possible degradation of matrix extracellular elastics components, since the mRNA levels to matrix's metaloproteases (MMP 2 and 9), enzymes responsible for degrade the elastic matrix, are increased.

Wild type and homozygous FBN-1 cells were isolated and cultivated. After 4 days of confluence, the culture medium was collected and, after ammonium sulfate precipitation, proteins were evaluated by gel-filtration chromatography, SDS-page electrophoresis and by Western blot analysis. As shown in Image 1, it was not possible to visualize the medium proteins, except albumin (69 kDa), the major ingredient of Bovine Fetal Serum (BFS), used in the treatment of the culture medium.

The proteins contained in the culture medium of deficient fibrillin- 1 cells after 4 days of confluence, degradation products of fiber elastics, may have low molecular weight, so it is possible that it was not precipitated with the ammonium sulfate's used concentrations. The electrophoresis SDS-page $10 \%$ of acrylamide may not included the molecular weight of those proteins.

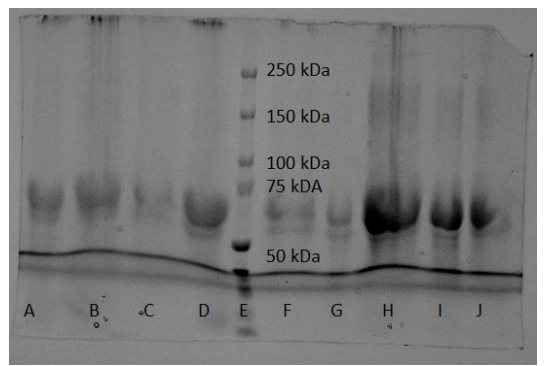

Image 1. Electrophoresis SDS-page 10\%of acrylamide of culture medium of wild type $(\mathrm{A}, \mathrm{C}$ and $\mathrm{H})$ and homozygous FBN1 (B, G and I) cells after 4 days of confluence, compared with culture medium DMEM (D, $F$ and $J$ ) in different concentrations of ammonium sulfate: $60 \%(A, B$ and $\mathrm{D}), 65 \%(\mathrm{C}, \mathrm{F}$ and $\mathrm{G})$ and $70 \%(\mathrm{H}, \mathrm{I}$ and $\mathrm{J})$.

\section{Conclusions}

In conclusion, the results presented in this study implicate that deficient fibrillin-1 cells have decreased deposition of elastic components. Different deposition pattern may be result of decreased deposition and/or degradation of the extracellular matrix, because of the presence of increased matrix's metaloproteases mRNA. It was not possible to verify degradation products of elastic fibers in those cells by the methods used in the analysis, and it may be necessary to use higher concentrations of ammonium sulfate for protein precipitation and higher acrylamide electrophoresis concentration.

\section{Acknowledgement}

This work was supported by CNPq.

\footnotetext{
Neptune, E. R., Frischmeyer, et al (2003). Dysregulation of TGF-beta activation contributes to pathogenesis in Marfan syndrome. Nat Genet 33, 407-411

Matt, P., e t a 1 (2008). Recent advances in understanding Marfan syndrome: should we now treat surgical patients with losartan? J Thorac Cardiovasc Surg 135, 389-394'
} 